\title{
APOBEC3B is expressed in human glioma, and influences cell proliferation and temozolomide resistance
}

\author{
CHRISTINA SCHMITT ${ }^{1}$, RALPH LUCIUS ${ }^{1}$, MICHAEL SYNOWITZ ${ }^{2}$, \\ JANKA HELD-FEINDT $^{2 *}$ and KIRSTEN HATTERMANN ${ }^{1 *}$ \\ ${ }^{1}$ Institute of Anatomy, University Kiel, D-24098 Kiel; ${ }^{2}$ Department of Neurosurgery, \\ University Medical Center Schleswig-Holstein UKSH, D-24105 Kiel, Germany
}

Received February 15, 2018; Accepted August 1, 2018

DOI: $10.3892 / o r .2018 .6698$

\begin{abstract}
Highly malignant gliomas are characterized by pronounced intra- and intertumoral heterogeneity. On the genetic level, this heterogeneity may be caused by spontaneous mutation events, but recent studies have reported distinct mutational signatures that may be caused by an enzyme family with cytidine desaminase activity, the apolipoprotein B mRNA editing enzyme catalytic polypeptide-like (APOBEC) proteins. Among these, APOBEC3B contributes to tumor progression in a variety of types of tumor, including breast cancer. In the present study, the expression of APOBEC3B was detected at the mRNA and protein levels in solid human glioma tissue and human glioma cell lines. In vitro, treatment with temozolomide, the most commonly used chemotherapeutic in glioma therapy, induced APOBEC 3B expression. Furthermore, the knockdown of APOBEC3B by clustered regularly interspaced short palindromic repeats/CRISPR associated protein 9 resulted in reduced proliferation and enhanced chemosensitivity of glioma cells. Thus, APOBEC3B contributes to glioma progression and may be a future target for therapeutic intervention.
\end{abstract}

\section{Introduction}

Glioblastomas are highly malignant brain tumors that have a poor prognosis for patients despite advanced surgery and

Correspondence to: Dr Kirsten Hattermann, Institute of Anatomy, University Kiel, 40 Olshausen Road, D-24098 Kiel, Germany

E-mail:k.hattermann@anat.uni-kiel.de

*Contributed equally

Abbreviations: APOBEC, apolipoprotein B mRNA editing enzyme, catalytic polypeptide-like; Cq, cycle of threshold; GBM, glioblastoma multiforme; GFAP, glial fibrillary acidic protein; HIV, human immunodeficiency virus; ICC, immunocytochemistry; RT-qPCR, reverse transcription-quantitative polymerase chain reaction

Key words: glioma, APOBEC3B, heterogeneity, tumor evolution, chemoresistance subsequent aggressive treatment with combined radiotherapy and chemotherapy (1). Over 90 years ago, Bailey and Cushing established the term glioblastoma multiforme (2) for the astrocytoma World Health Organization (WHO) grade IV, paying tribute to the pronounced heterogeneity of this type of tumor. This heterogeneity has been observed and investigated in a variety of aspects of glioblastoma biology, and attributed to the differential response of patients to therapy $(3,4)$. Cell cycle control and DNA repair mechanisms have been identified as key aspects at the evolutionary origin of the pronounced interand intratumoral genetic heterogeneity of gliomas, amongst others $(5,6)$. However, a recent study indicated an important role of the apolipoprotein B mRNA editing enzyme catalytic polypeptide-like (APOBEC) enzyme family in genetic instability and heterogeneity, as specific patterns of DNA mutagenesis have been observed in various types of human tumors (7). The evolutionarily highly conserved APOBEC enzyme family members have cytidine desaminase activity, by which they exert post-transcriptional editing of the mRNA by converting cytosine to uracil; for example, in the mRNA of apolipoprotein B and neurofibromin (8). Certain APOBEC family members also support the innate immune system by editing the viral genome, particularly the genome of retroviruses like the human immunodeficiency virus HIV (9). In cancer, APOBEC family members are key players in the induction of cancer evolution and heterogeneity (10). In particular, APOBEC3B has been identified as the major cause for typical mutagenesis patterns, for example in breast (11), bladder, cervix, head and neck, and lung cancer (12). Although a recent study has addressed a putative APOBEC enzyme-caused mutational signature in gliomas (13), to the best of our knowledge, there is no information available on the APOBEC 3B protein in human gliomas. Thus, the present study investigated the expression and functional role of APOBEC 3B in human glioma tissue and cell lines.

\section{Materials and methods}

Tissue and cell lines. Glioma samples of different malignancy grades were obtained at the Department of Neurosurgery, University Medical Center Schleswig-Holstein UKSH (Kiel, Germany) in accordance with the Declaration of Helsinki (1975) with approval from the Ethics Committee of the University of 
Kiel (Kiel, Germany) and after written informed consent was obtained from the donors (file reference, D536/15). Diagnosis was established by a pathologist and malignancy grades were determined according to the WHO classification system. Non-neoplastic brain tissue samples were obtained from the Institute of Legal Medicine, University Kiel. Glioma cell lines LN229, A172, T98G and U251MG were purchased from the European Collection of Cell Cultures (Salisbury, UK) or the American Type Culture Collection (Manassas, VA, USA) and cultured in Dulbecco's modified Eagle's medium (DMEM) plus $10 \%$ fetal bovine serum (FBS) (Invitrogen; Thermo Fisher Scientific, Inc., Waltham, MA, USA). Cells were routinely checked for Mycoplasma contamination using bisbenzimide staining, and for identity/purity by short tandem repeat profiling at the Department of Forensic Medicine, University of Kiel where the Powerplex HS Genotyping kit (Promega Corporation, Madison, WI, USA) and a 3500 Genetic analyzer (Thermo Fisher Scientific, Inc.) were employed.

Cell stimulations and clustered regularly interspaced short palindromic repeats (CRISPR)/CRISPR associated protein 9 (Cas9) silencing. In order to analyze the regulation of APOBEC3B expression, 5.0x10 ${ }^{4} \mathrm{LN} 229$ cells/well were seeded in six-well plates or $2.0 \times 10^{4} \mathrm{LN} 229$ cells were seeded on glass cover slips and grown for $24 \mathrm{~h}$ in DMEM with $10 \%$ FBS. Cells were then treated with $100 \mu \mathrm{g} / \mathrm{ml}$ temozolomide or a corresponding amount of the solvent DMSO for a total of 10 days, during which the media were changed every 2-3 days, and the DMSO-treated cells were detached and seeded again at the same densities on day 6. After 10 days, cells were harvested for mRNA isolation and reverse transcription-quantitative polymerase chain reaction (RT-qPCR), or the glass slides with cells were used for immunocytochemistry (ICC).

For CRISPR/Cas9 silencing, 5.0x10 ${ }^{4}$ LN229 cells/well were seeded in six-well plates and transfected with $1 \mu \mathrm{g}$ of the APOBEC3B Double Nickase plasmid (cat. no. sc-401700-NIC) or the corresponding Double Nickase Control plasmid (cat. no. sc-437281) using the UltraCruz Transfection reagent (all from Santa Cruz Biotechnology, Inc., Dallas, TX, USA) for $24 \mathrm{~h}$. Following the manufacturer's protocol, selection was performed with puromycin (Invitrogen; Thermo Fisher Scientific, Inc.), and clones were selected and analyzed using RT-qPCR, ICC and western blot analysis.

$R T-q P C R$. For mRNA expression analysis, cells were harvested and tissues were homogenized using QIAzol Lysis reagent (Qiagen GmbH, Hilden, Germany) and total RNA was isolated according to the manufacturer's protocol. Genomic DNA was digested by RNase-free DNase (1 U/ $\mu \mathrm{l}$; Promega Corporation) and cDNA was synthesized using RevertAid ${ }^{\mathrm{TM}} \mathrm{H}$ Minus M-MuLV Reverse Transcriptase (200 U/ $\mu$ l; Thermo Fisher Scientific, Inc.) according to the manufacturer's protocol. RT-qPCR was performed as previously described (14) using the following TaqMan Primer probes (Applied Biosystems; Thermo Fisher Scientific, Inc.): hGAPDH (Hs99999905_m1) and hAPOBEC3B (Hs00358981_m1). Samples were analyzed in duplicates and cycle threshold $(\mathrm{Cq})$ values were measured with the ABI PRISM 7500 Sequence Detection system (Applied Biosystems; Thermo Fisher Scientific, Inc.). The thermocycling conditions were as follows: $2 \mathrm{~min}$ at $50^{\circ} \mathrm{C}$ and
$10 \min 95^{\circ} \mathrm{C}$, followed by 40 cycles of $15 \mathrm{sec}$ at $95^{\circ} \mathrm{C}$ and $1 \mathrm{~min}$ at $60^{\circ} \mathrm{C}$. Cq values were used to calculate $\Delta \mathrm{Cq}$ values [Cq(APOBEC3B) - Cq(GAPDH)]. Due to logarithmic reaction process, a $\Delta \mathrm{Cq}$ value of 3.33 corresponds to one magnitude lower gene expression compared with that of GAPDH. For stimuli-induced mRNA regulation, $\Delta \Delta \mathrm{Cq}$ values were calcu-

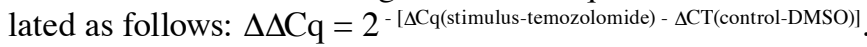
For statistical analysis, undetectable samples were calculated as the anticipated maximum cycle number-Cq (GAPDH).

Immunohistochemistry and ICC. Immunohistochemistry was performed as previously described (15). Briefly, cryostat sections from three different fresh-frozen glioblastoma samples were fixed in ice-cold acetone/methanol (1:1) for $10 \mathrm{~min}$ at room temperature and washed with Tris-buffered saline with $0.1 \%$ Tween (TBS-T). The samples were then blocked for autofluorescence with $1 \%$ Sudan black (in $70 \%$ ethanol) for $1 \mathrm{~h}$ at room temperature and for unspecific binding with $0.5 \%$ glycine $/ 0.5 \%$ bovine serum albumin (SERVA Electrophoresis GmbH, Heidelberg, Germany) in TBS and incubated with the primary antibodies overnight at $4^{\circ} \mathrm{C}$. Subsequently, the samples were washed with TBS-T and incubated with the secondary antibodies for $1 \mathrm{~h}$ at $37^{\circ} \mathrm{C}$. The samples then were washed again with TBS-T, incubated with 4',6-diamidino-2-phenylindole (DAPI; 1:30,000; Invitrogen; Thermo Fisher Scientific, Inc.) for $30 \mathrm{~min}$ at room temperature to counterstain the nuclei, washed with TBS-T, and embedded with Shandon ${ }^{\mathrm{TM}}$ Immu-Mount $^{\mathrm{TM}}$ (Thermo Fisher Scientific, Inc.). The primary antibodies were as follows: Anti-human APOBEC3B (rabbit polyclonal; 1:100; cat. no. TA349029; OriGene Technologies, Inc., Rockville, MD, USA); anti-human glial fibrillary acidic protein (GFAP; mouse monoclonal; 1:100; cat. no. M0761; Dako; Agilent Technologies, Inc., Santa Clara, CA, USA), anti-human made in borstel-1 (MIB-1; mouse monoclonal; 1:100; cat. no. M7240; Dako; Agilent Technologies, Inc.) and anti-human platelet endothelial cell adhesion molecule (Pecam-1; goat polyclonal; 1:100; cat. no. sc-1506; Santa Cruz Biotechnology, Inc.). The secondary antibodies were as follows: Donkey anti-rabbit IgG Alexa Fluor 555 (cat. no. A-31572); donkey anti-mouse IgG Alexa Fluor 488 (cat. no. A-21202); and donkey anti-goat IgG Alexa Fluor 488 (cat. no. A-11055) (all 1:1,000; Invitrogen; Thermo Fisher Scientific, Inc.).

For immunocytochemical analysis, cells were seeded on glass cover slips $\left(2.0 \times 10^{4}\right.$ cells/cover slip) and grown for 1 day (or for the subsequent treatment period). The cells were fixed with ice-cold acetone-methanol for $10 \mathrm{~min}$ at room temperature, blocked with $0.5 \%$ glycine $/ 0.5 \%$ bovine serum albumin in PBS for $1 \mathrm{~h}$ at room temperature and incubated with anti-human APOBEC3B (rabbit polyclonal; 1:100) overnight at $4{ }^{\circ} \mathrm{C}$. The cells were then washed with $\mathrm{PBS}+0.1 \%$ Tween (PBS-T; 3X) and incubated with donkey anti-rabbit IgG Alexa Fluor 555 (Invitrogen; Thermo Fisher Scientific, Inc.) at $37^{\circ} \mathrm{C}$ for $1 \mathrm{~h}$. Subsequently, the cells were washed with PBS-T $(3 \mathrm{X})$, the nuclei were counterstained with DAPI for $30 \mathrm{~min}$ at room temperature, and the slips were embedded using Immu-Mount.

The slides were viewed and imaged using a Zeiss Axiovert 200 M fluorescence microscope (Zeiss AG, Oberkochem, Germany) at x400 and x630 magnification. In order to compare 
differences in the expression of APOBEC3B, equal exposure times were used.

Western blot analysis. For western blot analysis, cells were lysed (50 mM Tris, $100 \mathrm{mM} \mathrm{NaCl}, 5 \mathrm{mM}$ EDTA, $1 \%$ Triton X-100, pH 7.8), and protein content was measured using the Bradford protein assay. Protein content was adjusted to $5 \mu \mathrm{g} / 20 \mu \mathrm{l}$ with sample buffer (10\% glycerin, $0.4 \%$ SDS, $50 \mathrm{mM}$ dithiothreitol in $75 \mathrm{mM}$ Tris), and samples were separated by SDS-PAGE ( $10 \%$ acrylamide gels, $5 \mu \mathrm{g} /$ lane) and blotted onto a polyvinylidene fluoride membrane. The membrane was blocked with 5\% skim milk powder in TBS-T for unspecific binding for $1 \mathrm{~h}$ at room temperature and incubated with anti-human APOBEC3B (rabbit polyclonal; 1:250) overnight at $4^{\circ} \mathrm{C}$. Subsequently, the membrane was washed with TBS-T (3X) and incubated with horseradish peroxidase-conjugated secondary antibody overnight at $4^{\circ} \mathrm{C}$ (goat anti-rabbit; 1:30,000; cat. no. sc-2004; Santa Cruz Biotechnology, Inc.). The membrane was then washed again with TBS-T, followed by chemoluminescence signal detection (Immobilon; Merck KGaA, Darmstadt, Germany) using a Peqlab Fusion camera (Peqlab Biotechnologie GmbH, Erlangen, Germany). Blots were reprobed following stripping (ReBlot Plus Strong Antibody Striping solution; Merck KGaA) for GAPDH (primary antibody mouse anti-human; 1:250; cat. no. sc-47724; goat anti-mouse horseradish peroxidase-conjugated secondary antibody; 1:30,000; cat. no. sc-2005; from Santa Cruz Biotechnology, Inc.) in order to compare loading using the same protocol aforementioned.

Proliferation assay. In order to compare the proliferation of LN229 APOBEC3B CRISPR/Cas9 knockdown clones with that of control clones, $2.5 \times 10^{4}$ cells/well were seeded in 6-well plates in triplicates, and cultured for 24, 48, 72 and $96 \mathrm{~h}$. Cells were then washed with PBS, harvested, and analyzed using a CyQUANT $^{\circledR}$ cell proliferation assay (Thermo Fisher Scientific, Inc.) according to the manufacturer's protocol. As an internal standard, $2.5 \times 10^{4}$ cells (APOBEC3B and control clones) were lysed and measured, and fluorescence signals were normalized to these samples.

Caspase 3/7 assay. In order to analyze the influence of APOBEC $3 B$ on the sensitivity of cells to temozolomide, the most common chemotherapeutic agent used in glioblastoma therapy, 3.0x10 $0^{5}$ APOBEC 3B clone cells or control clone cells were seeded in T25 flasks, and cultured for $24 \mathrm{~h}$. Subsequently, the medium was replaced by fresh DMEM with $10 \%$ FBS and different concentrations of temozolomide (or an equal volume of DMSO as a solvent control), and the cells were treated for a further $48 \mathrm{~h}$. Caspase 3/7 activity was measured as previously described (16).

Statistical analysis. All values are presented as the mean \pm standard deviation. Expression values from tumor samples were compared with non-neoplastic brain tissue samples using one-way analysis of variance (ANOVA) with Dunnet's multiple comparison post hoc test. To analyze induction of APOBEC3B expression, a paired Student's t-test was used. For statistical analysis of biological effects of APOBEC3B silencing (proliferation and caspase 3/7 assays) one-way ANOVA with Bonferroni's multiple comparison post hoc test was used to compare control clones with APOBEC3B knockdown clones at different time points (proliferation) or upon different temozolomide treatment doses (caspase 3/7 assay). $\mathrm{P}<0.05$ was considered to indicate a statistically significant difference.

\section{Results}

Expression and drug-induced regulation of $A P O B E C 3 B$ in gliomas. As the initial step, the expression and cellular localization of APOBEC3B in solid human astrocytoma samples of different malignancy grades, as well as in commercial glioblastoma cell lines, were investigated. Additionally, the regulation of the amounts of APOBEC $3 \mathrm{~B}$ mRNA and protein was analyzed in temozolomide-treated glioblastoma cells.

APOBEC3B was expressed in all of the glioma samples in considerable amounts (mean $\Delta \mathrm{Cq}$ value range, 8.0-9.0 for astrocytoma grade I-IV; grade IV astrocytoma is also known as glioblastoma). Compared with the normal brain control samples, APOBEC3B was significantly overexpressed in glioblastoma tissue. The mean $\Delta \mathrm{Cq}$ values were 11.2 for the normal brain samples, and 8.4, 9.1, 8.2 and 8.0 for grade I-IV astrocytoma samples, respectively (Fig. 1, left). A $\Delta \mathrm{Cq}$ value of 3.33 corresponds to one magnitude lower gene expression compared with that of the reference marker GAPDH. In the glioblastoma samples, APOBEC3B was expressed in GFAP-positive astrocytic tumor regions and was detected near to Pecam-1-positive tumor vessels, but was not co-stainable with MIB-1 (antigen Ki-67), which is a marker of active proliferating cells (Fig. 1, right).

With the exception of the U251MG cell line, APOBEC3B was evidently detectable in the glioblastoma cell lines investigated (A172, LN229, T98G and U251MG) at the transcriptional level, as determined using RT-qPCR (Fig. 2, left). The mean $\Delta$ Cq values were as follows: $8.4 \pm 0.7$ (A172), $6.7 \pm 0.2$ (LN229), $6.8 \pm 1.1(\mathrm{~T} 98 \mathrm{G})$ and $18.5 \pm 3.0(\mathrm{U} 251 \mathrm{MG})(\mathrm{n}=4$ independent experiments). Furthermore, APOBEC3B expression was evaluated at the translational level using ICC in the LN229, T98G and U251MG cell lines ( $\mathrm{n}=2$ independent experiment; Fig. 2, right). When stimulating LN229 glioblastoma cells with temozolomide ( $100 \mu \mathrm{g} / \mathrm{ml}$ for 10 days), the standard chemotherapeutic drug used in glioblastoma treatment, APOBEC3B mRNA expression was increased up to 9-fold (range, 3.5 to 9.2-fold; mean, 6.1 \pm 1.9 -fold; Fig. 3, left; $\mathrm{n}=8$ independent experiments; $\mathrm{P}<0.05$ ) of that in the DMSO control group. In accordance with this, APOBEC $3 \mathrm{~B}$ protein was detectable in markedly higher amounts in the temozolomide-treated LN229 cells compared with those of the DMSO control-treated cells (Fig. 3, right; $n=2$ independent experiments).

Biological functions of $A P O B E C 3 B$ in glioblastoma. In order to investigate the biological functions of APOBEC3B in glioblastoma, stable APOBEC3B CRISPR/Cas9 knockdown LN229 clones were established. The knockdown efficiency was confirmed using RT-qPCR, western blot analysis and ICC. All methods demonstrated clearly diminished levels of APOBEC3B expression at the mRNA and protein levels. At the mRNA level $(n=3)$ APOBEC3B expression was reduced to $1.7 \pm 0.8 \%$ in comparison with 

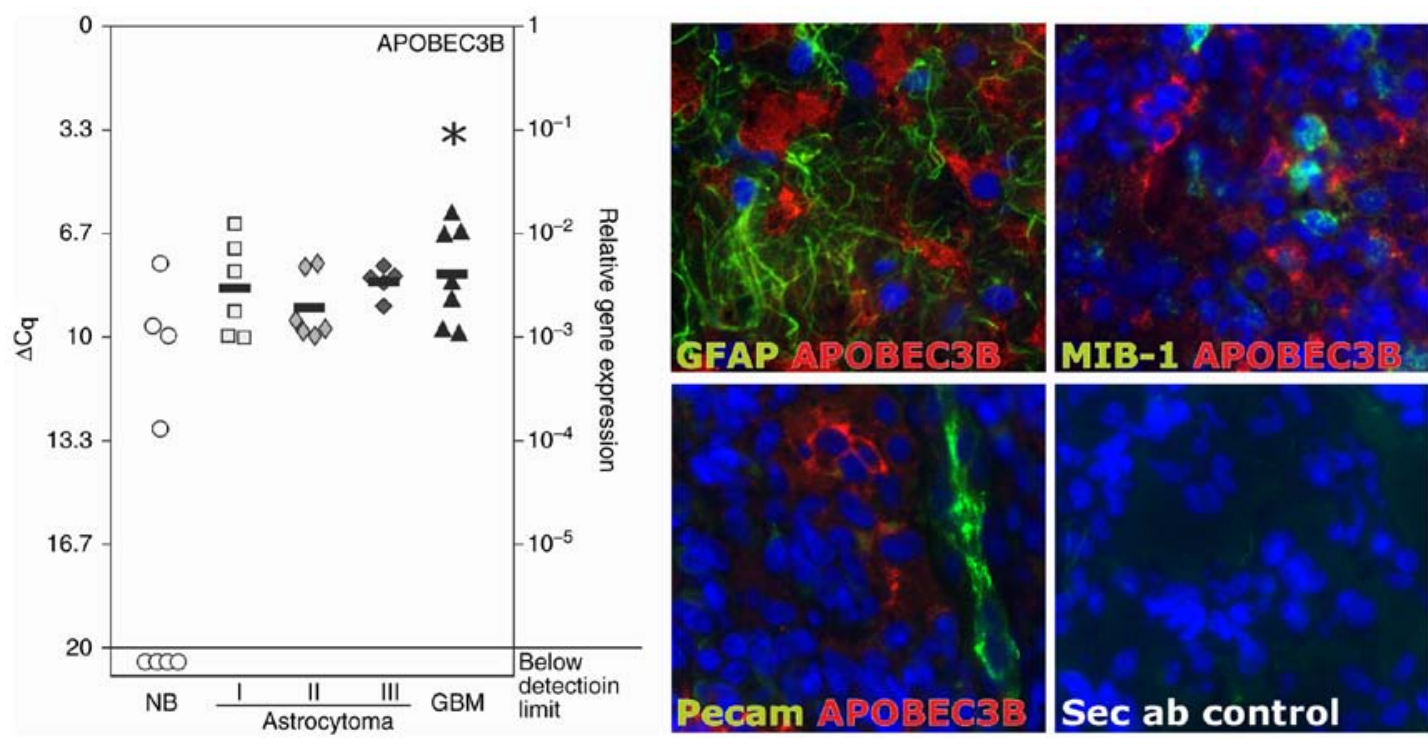

Figure 1. Expression of APOBEC3B in solid human gliomas. Left: Transcription of APOBEC3B was analyzed in eight NB, six grade I astrocytoma, six grade II astrocytoma, five grade III astrocytoma and seven grade IV astrocytoma/GBM using RT-qPCR. In all of the investigated glioma samples, APOBEC3B mRNA was clearly detectable. In the grade IV tumors, the levels of mRNA expression were significantly higher compared with those in the non-neoplastic brain tissues. Black bars indicate mean values and a lower $\triangle \mathrm{Cq}$ value of 3.33 corresponds to a magnitude higher expression. In a number of the non-neoplastic brain tissue samples, APOBEC3B could not be detected (below detection limit); thus, a mean bar could not be included. Right: Expression and localization of APOBEC3B were analyzed using immunohistochemistry in the human glioblastoma samples. APOBEC3B (red) was detected on the protein level in GFAP-positive tumor regions (green), frequently in small cell clusters and in proximity to blood vessels (stained by Pecam-1; green), and co-staining with MIB-1, a marker of proliferating cells, was not evident. For secondary antibody controls, primary antibodies were respectively omitted. Representative examples of three individual glioblastoma samples are shown at x630 magnification. " $\mathrm{P}<0.05$, one-way analysis of variance followed by Dunnet's multiple comparison test. APOBEC3B, apolipoprotein B mRNA editing enzyme catalytic polypeptide-like protein 3B; Cq, cycle of threshold; GBM, glioblastoma multiforme; GFAP, glial acidic fibrillary protein; MIB-1, made in Borstel-1; NB, normal brain; Pecam-1, platelet endothelial cell adhesion molecule; Sec ab, secondary antibody control.
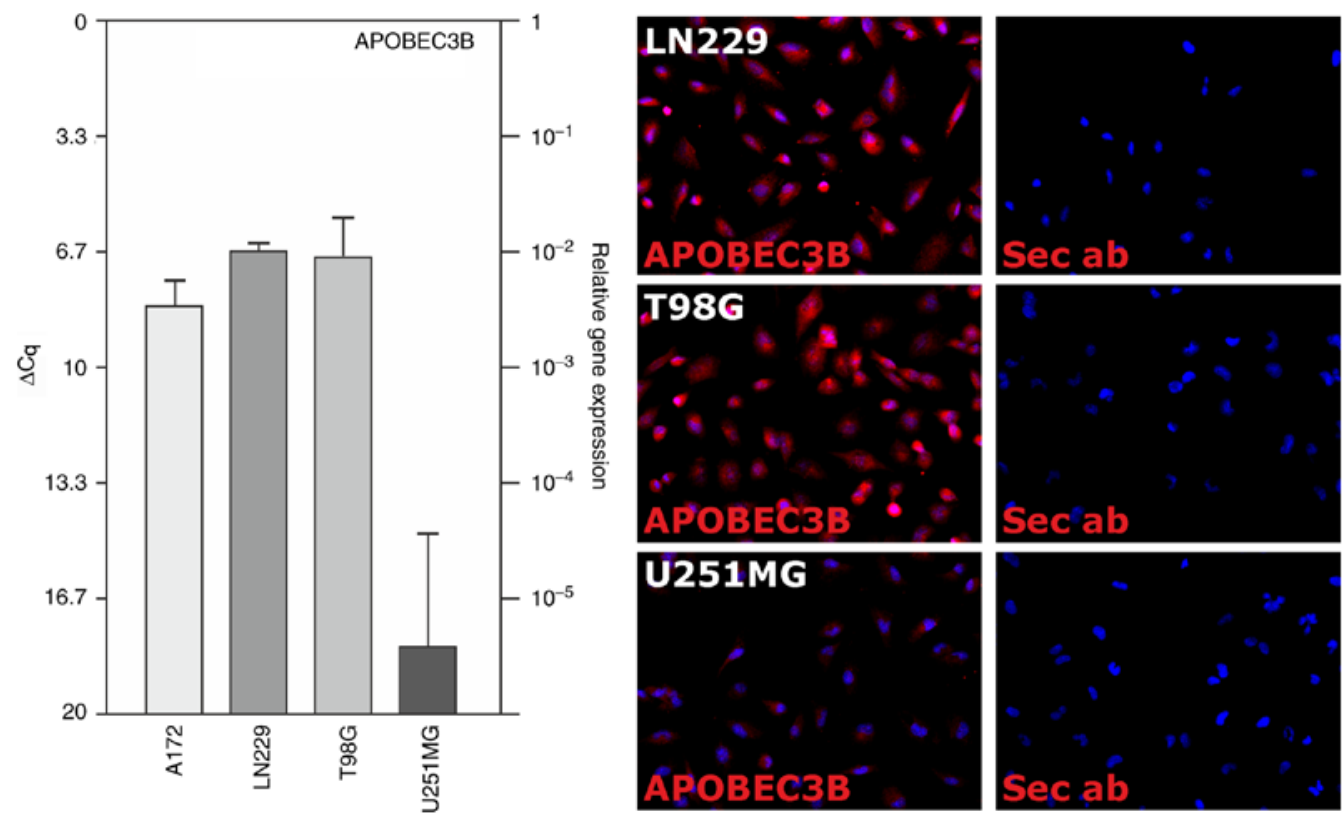

Figure 2. Expression of APOBEC3B in human glioblastoma cell lines. APOBEC3B expression was analyzed on the mRNA (RT-qPCR, left) and protein (ICC, right) levels in human glioblastoma cell lines. Clearly detectable levels of mRNA expression were observed in the A172, LN229 and T98G cells, while APOBEC3B was hardly detected in the U251MG cells. At the protein level, APOBEC3B expression was exemplarily analyzed in the LN229, T98G and U251MG cells. Data are presented as the mean $\Delta$ Cq values \pm standard deviation from $n=4$ individual RNA preparations and exemplary results from $n=2$ individual immunostainings at $\mathrm{x} 400$ magnification. APOBEC3B, apolipoprotein B mRNA editing enzyme catalytic polypeptide-like protein 3B; Cq, cycle of threshold; Sec ab, secondary antibody control; RT-qPCR, reverse transcription-quantitative polymerase chain reaction; ICC, immunocytochemistry.

the corresponding control CRISPR/Cas9 clones (data not shown). At the protein level, evident reduced signals were observed in the western blot experiments (Fig. 4A) and no APOBEC3B signals in the APOBEC3B CRISPR/Cas9 knockdown LN229 clones were identified by ICC (equal exposure times, Fig. 4A). 


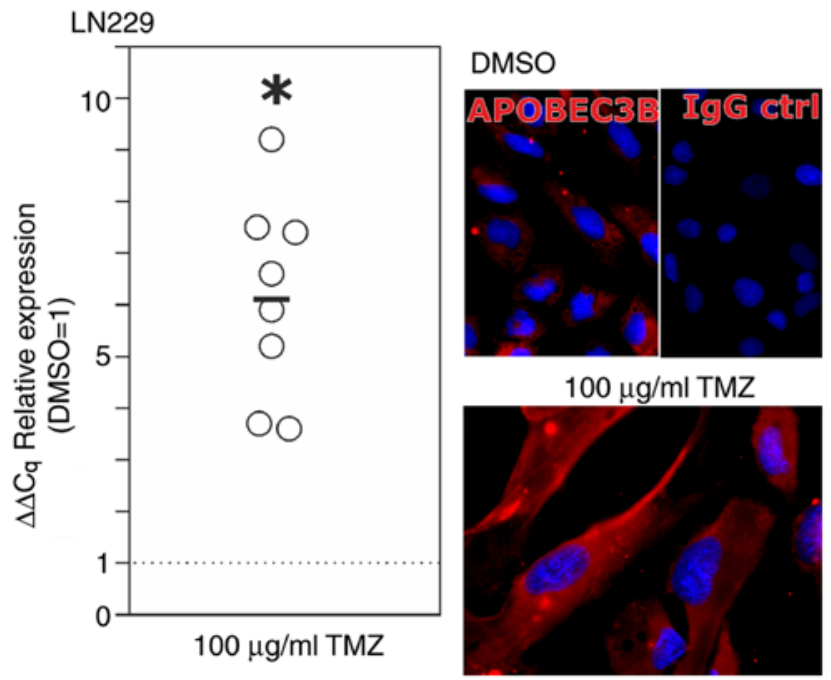

Figure 3. Regulation of APOBEC3B upon TMZ treatment in vitro. LN229 glioma cells were treated for 10 days with $100 \mu \mathrm{g} / \mathrm{ml} \mathrm{TMZ}$ or equal volumes of the solvent DMSO as a control. Expression analysis using RT-qPCR (left, circles indicate independent experiments, black bar indicates mean value from n=8 independent experiments, "P<0.05; Student's two-tailed t-test) and ICC (representative examples of $\mathrm{n}=2$ independent stimulations) demonstrated a significant induction (mean, 6.1-fold) of APOBEC3B upon TMZ treatment. For the microscopic images, the same magnifications (x630) and exposure times were used. APOBEC3B, apolipoprotein B mRNA editing enzyme catalytic polypeptide-like protein 3B; Cq, cycle of threshold; DMSO, dimethylsulfoxide; IgG ctrl, IgG control; TMZ, temozolomide; RT-qPCR, reverse transcription-quantitative polymerase chain reaction; ICC, immunocytochemistry.
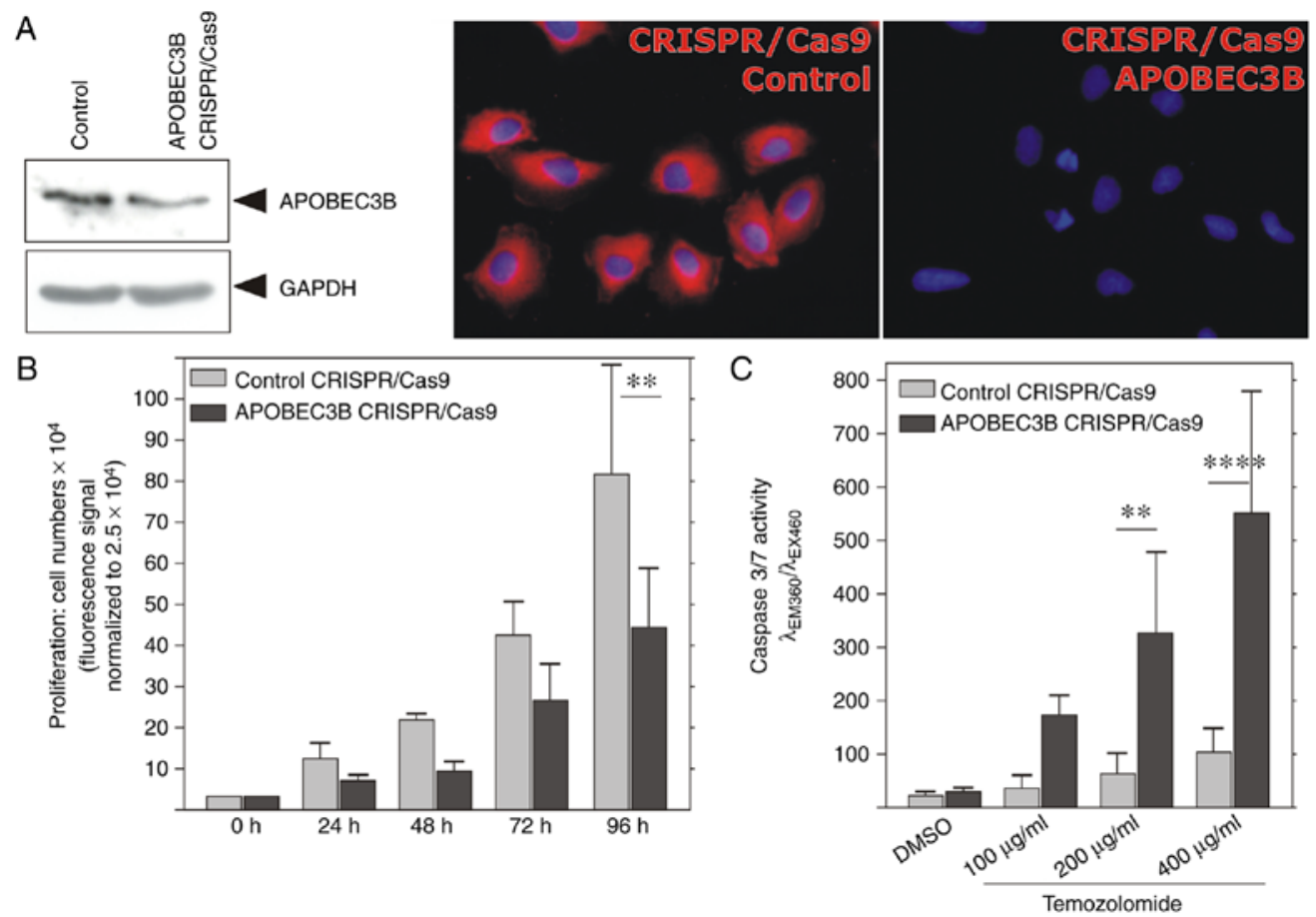

Figure 4. Knockdown of APOBEC3B reduces proliferation and enhances sensitivity of cells to TMZ. (A) In LN229-derived CRISPR/Cas9 knockdown clones of APOBEC3B, expression levels of APOBEC3B were markedly reduced compared with the control clones, as shown by western blotting (detection of GAPDH served as the loading control) and ICC. For the microscopic images, the same magnifications (x400) and exposure times were used. (B) LN229-derived CRISPR/Cas9 knockdown clones of APOBEC3B proliferated less compared with the corresponding control clones in a time period of 48-96 h, as detected by measurement of the DNA content. ${ }^{* *} \mathrm{P}<0.01$, one-way analysis of variance followed by Bonferroni's multiple comparison test. (C) When exposed to different concentrations of TMZ for $48 \mathrm{~h}, \mathrm{LN} 229$ APOBEC3B-knockdown cells exhibited significantly higher levels of caspase 3/7 activity compared with those of the corresponding control clones upon 200 and $400 \mu \mathrm{g} / \mathrm{ml} \mathrm{TMZ}$ treatment. ${ }^{* *} \mathrm{P}<0.01$ and ${ }^{* * * *} \mathrm{P}<0.0001$, one-way analysis of variance followed by Bonferroni's multiple comparison test. The levels of basic caspase 3/7 activity in DMSO-treated cells were not significantly altered in the APOBEC3B CRISPR/Cas9 clones, compared with those of the control clones. Data are presented as the mean values \pm standard deviation of $n=3$ independent experiments. APOBEC $3 \mathrm{~B}$, apolipoprotein B mRNA editing enzyme catalytic polypeptide-like protein 3B; GAPDH, glyceraldehyde 3-phosphate dehydrogenase; TMZ, temozolomide; CRISPR, clustered regularly interspaced short palindromic repeats; Cas9, CRISPR associated protein 9.

Investigation of the proliferative potential of APOBEC3B CRISPR/Cas9 knockdown LN229 clones for $\leq 96 \mathrm{~h}$ compared with that of their control-transfected counterparts revealed that LN229 clones with diminished APOBEC3B 
expression were characterized by a reduction in proliferation levels (Fig. 4B; $\mathrm{n}=3$ independent experiments; $\mathrm{P}<0.01$ at $96 \mathrm{~h}$ ). Furthermore, as demonstrated by caspase 3/7 activity, APOBEC3B CRISPR/Cas9 knockdown LN229 clones exhibited significantly higher levels of sensitivity to temozolomide treatment compared with those of the controls (Fig. 4C). Increasing concentrations of temozolomide $(100-400 \mathrm{mg} / \mathrm{ml}$; $48 \mathrm{~h}$ ) yielded increasing levels of caspase 3/7 activity in the control cells; however, in the APOBEC3B knockdown clones, the concentration-dependent induction of the caspase 3/7 activity was significantly more prominent $(200 \mu \mathrm{g} / \mathrm{ml} \mathrm{TMZ}$, $\mathrm{P}<0.01 ; 400 \mu \mathrm{g} / \mathrm{ml} \mathrm{TMZ}, \mathrm{P}<0.0001)$. The caspase $3 / 7$ activity in DMSO-treated samples was not significantly different between the APOBEC3B and control CRISPR/Cas9 clones. Thus, APOBEC3B mediates proliferative and anti-apoptotic effects in human glioblastoma cells.

In summary, the APOBEC3B enzyme was evidently detectable in human glioma samples of different malignancy grades, and was observable in astrocytic tumor regions and nearby tumor vessels. Furthermore, the APOBEC3B enzyme was inducible upon temozolomide treatment, and mediated proliferative and anti-apoptotic effects in human glioblastoma cells.

\section{Discussion}

A previous study has demonstrated that members of the APOBEC family may cause a characteristic pattern of mutations in a several types of cancer (7). Apart from their physiological role in mRNA editing, this enzyme family has important functions in the innate immune response to retroviruses and retrotransponsons, and may also contribute to tumor heterogeneity and progression via its DNA-desaminase activity $(9,10)$. In the present study, it was demonstrated that APOBEC3B, a member of the APOBEC family, was overexpressed in glioblastoma (WHO grade IV), and was identified in astrocytic tumor regions and nearby tumor vessels. To date, APOBEC3B has only been detected in human leptomeninges and meningioma, to the best of our knowledge (17). However, Talagas et al (18) reported that certain oligodendroglial intracranial tumors that do not exhibit $1 \mathrm{p} / 19 \mathrm{q}$ deletions are characterized by various other mutations, including a homozygous deletion at 22q13.1 (APOBEC3B gene). To the best of our knowledge, there is no data regarding on the role of APOBEC3B in astroglial tumors to date. Focusing on another APOBEC family member in astroglial tumors, a recent study indicated an important role for APOBEC 3G in mesenchymal glioma, a highly malignant brain tumor type (19). APOBEC3G knockdown attenuated the proliferation and invasion of glioblastoma cell lines by influencing the transforming growth factor (TGF)- $\beta$-signaling pathway via smad2, which resulted in decreased expression levels of proteins, including thrombospondin-1 and matrix-metalloproteinase-2 (19). However, the exact mechanism by which APOBEC $3 \mathrm{G}$ regulates the TGF- $\beta$ pathway remains unclear. Notably, Wang et al (20) revealed that APOBEC3G is upregulated in human astrocytoma (U87MG) cells by stimulation with different interferons, interleukin-1 and tumor necrosis factor. In contrast, other cytokines, including interleukin- 4 or -6 and particularly TGF- $\beta$ did not induce
APOBEC 3G expression. In the present study, expression of APOBEC3B was induced in cultured glioblastoma cells by temozolomide, the most commonly used chemotherapeutic in glioma therapy. Particularly in light of the proliferative and anti-apoptotic effects of APOBEC3B demonstrated in the present study, this induction may also influence the progression of glioma.

APOBEC3B is known to be involved in several types of cancer (12,21-27) and the results of the present study suggest that APOBEC3B may serve a more prominent role in brain cancer progression than previously assumed. Taking into account that Periyasamy et al (28) demonstrated that p53 regulates the expression of APOBEC $3 \mathrm{~B}$ in order to limit its potential mutagenic activity, the results of the present study are interesting. Glioblastoma are well known to be characterized by multiple mutations or allelic loss of the p53 tumor suppressor gene (3), and the A172, LN229, U251MG and T98G glioblastoma cell lines used in the present study exhibit missense or small frameshift mutations of the p53 gene [A172: p53 gene position 242, wt TGC, mut TTC (29); LN229: p53 gene position 164, wt AAG, mut GAG; U251MG: p53 gene position 273, wt CGT, mut CAT; and T98G: p53 gene position 237, wt ATG, mut ATA (30)]. As mutations or loss of the p53 gene result in elevated levels of APOBEC3B expression followed by increased levels of desaminase activity in cancer cells (28), the detectable amounts of APOBEC3B in glioblastoma cells may also be a consequence of p53 mutations. However, this paradigm does not apply absolutely as the U251MG cells were characterized by low levels of APOBEC3B expression (mean $\triangle \mathrm{Cq}, 18.5$ ) in the present study, and this cell line also contains p53 point mutations. APOBEC3B expression has been demonstrated to be regulated by other mechanisms, for example by Myb-related protein B (encoded by MYB proto-oncogene like 2), which binds to the APOBEC3B promoter causing its transactivation (31), or by the classical nuclear $(N F)-\kappa B$ signaling pathway (32). Three NF- $\kappa \mathrm{B}$ binding sites have been identified in the APOBEC3B promoter (32). In accordance with this, Leonard et al (33) demonstrated that protein kinase $\mathrm{C}$ activation causes the recruitment of RELB proto-oncogene $\mathrm{NF}-\kappa \mathrm{B}$ subunit (the gene encoding the transcription factor RelB), but not RELA proto-oncogene $\mathrm{NF}-\kappa \mathrm{B}$ subunit (the gene encoding the transcription factor p65), to the APOBEC3B promoter, indicating the involvement of the non-canonical $\mathrm{NF}-\kappa \mathrm{B}$ signaling.

With regards to its functional role, the results of the present study indicate that APOBEC3B mediates proliferative and anti-apoptotic effects in human glioblastoma cells, and, therefore, may promote glioma progression. However, a previous study reported that high levels of APOBEC $3 \mathrm{~B}$ expression causes hypersensitivity to small-molecule inhibitors that target the DNA damage response, suggesting that APOBEC3B overexpression also imparts targetable vulnerabilities upon cells (34).

In summary, APOBEC3B appears to serve a role in intracranial brain tumors, particularly in glioblastoma, contributing to glioma growth and heterogeneity by promoting the establishment of a mutational signature, and supporting glioma progression. APOBEC3B may therefore be a potential target for future therapeutic strategies against glioma. 


\section{Acknowledgements}

The authors would like to thank Ms. Judith Becker, Ms. Martina Burmester and Ms. Sonja Dahle (all from the Institute of Anatomy, University Kiel, Germany) for expert technical assistance.

\section{Funding}

The present study was supported by a grant from the Familie Mehdorn Foundation and by the Deutsche Forschungsgemeinschaft (grant no. RTG2154; projects 7 and 8).

\section{Availability of data and materials}

The raw datasets analyzed in this study are available from the corresponding author upon request.

\section{Authors' contributions}

RL, MS, JHF, CS and KH were involved in the conception and design of the study. CS performed the experiments and contributed to the data analysis, RL and MS provided essential materials. JHF and KH planned the experimental studies, analyzed the data and wrote the manuscript. All authors have critically revised and approved the manuscript.

\section{Ethics approval and consent to participate}

Glioma samples of different malignancy grades were obtained at the Department of Neurosurgery, University Medical Center Schleswig-Holstein UKSH (Kiel, Germany) in accordance with the Declaration of Helsinki (1975) with the approval of the Ethics Committee of the University of Kiel (Kiel, Germany) and after written informed consent was obtained from the donors (file reference, D536/15).

\section{Patient consent for publication}

Not applicable.

\section{Competing interests}

The authors declare that they have no competing interests.

\section{References}

1. Kreth FW, Thon N, Simon M, Westphal M, Schackert G, Nikkhah G, Hentschel B, Reifenberger G, Pietsch T, Weller M, et al; German Glioma Network: Gross total but not incomplete resection of glioblastoma prolongs survival in the era of radiochemotherapy. Ann Oncol 24: 3117-3123, 2013.

2. Bailey P and Cushing $\mathrm{H}$ : A classification of the tumours of the glioma group on a histogenetic basis: With a correlated study of prognosis. Medium 8vo. J.B. Lippincott Company, Philadelphia, London, and Montreal, with 108 illustrations, pp175, 1926. https://doi.org/10.1002/bjs.1800145540.

3. Brennan CW, Verhaak RG, McKenna A, Campos B, Noushmehr H, Salama SR, Zheng S, Chakravarty D, Sanborn JZ, Berman SH, et al: The somatic genomic landscape of glioblastoma. Cell 155: 462-477, 2013.

4. Hegi ME, Diserens AC, Godard S, Dietrich PY, Regli L, Ostermann S, Otten P, Van Melle G, de Tribolet $N$ and Stupp R: Clinical trial substantiates the predictive value of O-6-methylguanine DNA methyltransferase promoter treated with temozolamide. Clin Cancer Res 10: 1871-1874, 2004.
5. Dirks PB and Rutka JT: Current concepts in neuro-oncology: The cell cycle - A review. Neurosurgery 40: 1000-1013, 1997.

6. Stark AM, Doukas A, Hugo HH, Hedderich J, Hattermann K, Maximilian Mehdorn $\mathrm{H}$ and Held-Feindt J: Expression of DNA mismatch repair proteins MLH1, MSH2, and MSH6 in recurrent glioblastoma. Neurol Res 37: 95-105, 2015.

7. Roberts SA, Lawrence MS, Klimczak LJ, Grimm SA, Fargo D, Stojanov P, Kiezun A, Kryukov GV, Carter SL, Saksena G, et al: An APOBEC cytidine deaminase mutagenesis pattern is widespread in human cancers. Nat Genet 45: 970-976, 2013.

8. Wedekind JE, Dance GS, Sowden MP and Smith HC: Messenger RNA editing in mammals: New members of the APOBEC family seeking roles in the family business. Trends Genet 19: 207-216, 2003.

9. Sheehy AM, Gaddis NC, Choi JD and Malim MH: Isolation of a human gene that inhibits HIV-1 infection and is suppressed by the viral Vif protein. Nature 418: 646-650, 2002.

10. Swanton C, McGranahan N, Starrett GJ and Harris RS: APOBEC enzymes: Mutagenic fuel for cancer evolution and heterogeneity. Cancer Disc 5: 704-712, 2015.

11. Burns MB, Lackey L, Carpenter MA, Rathore A, Land AM, Leonard B, Refsland EW, Kotandeniya D, Tretyakova N, Nikas JB, et al: APOBEC3B is an enzymatic source of mutation in breast cancer. Nature 494: 366-370, 2013.

12. Burns MB, Temiz NA and Harris RS: Evidence for APOBEC3B mutagenesis in multiple human cancers. Nature Genet 45: 977-983, 2013.

13. Broad Institute TCGA Genome Data Analysis Center: Analysis of mutagenesis by APOBEC cytidine deaminase (P-MACD). Broad Institute of MIT and Harvard, 2015. doi: 10.7908/C12V2FBX.

14. Held-Feindt J, Hattermann K, Müerköster SS, Wedderkopp H, Knerlich-Lukoschus F, Ungefroren H, Mehdorn HM and Mentlein R: CX3CR1 promotes recruitment of human glioma-infiltrating microglia/macrophages (GIMs). Exp Cell Res 316: 1553-1566, 2010.

15. Hattermann K, Li G, Hugo HH, Mentlein R, Mehdorn HM and Held-Feindt J: Expression of the chemokines CXCL12 and CX3CL1 and their receptors in human nerve sheath tumors. Histol Histopathol 28: 1337-1349, 2013.

16. Hattermann K, Holzenburg E, Hans F, Lucius R, Held-Feindt J and Mentlein R: Effects of the chemokine CXCL12 and combined internalization of its receptors CXCR4 and CXCR7 in human MCF-7 breast cancer cells. Cell Tiss Res 357: 253-266, 2014.

17. Johnson MD, Reeder JE and O'Connell M: APOBEC3B expression in human leptomeninges and meningiomas. Oncol Lett 12: 5344-5348, 2016.

18. Talagas M, Marcorelles P, Uguen A, Redon S, Quintin-Roué I, Costa S, Férec C, Morel F, Hieu PD and De Braekeleer M: Identification of a novel population in high-grade oligodendroglial tumors not deleted on $1 \mathrm{p} / 19 \mathrm{q}$ using array $\mathrm{CGH}$. J Neurooncol 109: 405-413, 2012.

19. Wang Y, Wu S, Zheng S, Wang S, Wali A, Ezhilarasan R, Sulman EP, Koul D and Alfred Yung WK: APOBEC3G acts as a therapeutic target in mesenchymal gliomas by sensitizing cells to radiation-induced cell death. Oncotarget 8: 54285-54296, 2017.

20. Wang YJ, Wang X, Zhang H, Zhou L, Liu S, Kolson DL, Song L, Ye L and Ho WZ: Expression and regulation of antiviral protein APOBEC3G in human neuronal cells. J Neuroimmunol 206: 14-21, 2009.

21. Fanourakis G, Tosios K, Papanikolaou N, Chatzistamou I, Xydous M, Tseleni-Balafouta S, Sklavounou A, Voutsinas GE and Vastardis H: Evidence for APOBEC3B mRNA and protein expression in oral squamous cell carcinomas. Exp Mol Pathol 101: 314-319, 2016.

22. Jin Z, Han YX and Han XR: The role of APOBEC3B in chrondosarcoma. Oncol Rep 32: 1867-1872, 2014.

23. Leonard B, Hart SN, Burns MB, Carpenter MA, Temiz NA, Rathore A, Vogel RI, Nikas JB, Law EK, Brown WL, et al: APOBEC3B upregulation and genomic mutation patterns in serous ovarian carcinoma. Cancer Res 73: 7222-7231, 2013.

24. Olson ME, Harris RS and Harki DA: APOBEC enzymes as targets for virus and cancer therapy. Cell Chem Biol 25: 36-49, 2018.

25. Sasaki H, Suzuki A, Tatematsu T, Shitara M, Hikosaka Y, Okuda K, Moriyama S, Yano M and Fujii Y: APOBEC $3 B$ gene overexpression in non-small-cell lung cancer. Biomed Rep 2: 392-395, 2014.

26. Warren CJ, Westrich JA, Doorslaer KV and Pyeon D: Roles of APOBEC 3A and APOBEC3B in human papillomavirus infection and disease progression. Viruses 9: pii: E233, 2017. 
27. Wu PF, Chen YS, Kuo TY, Lin HH, Liu CW and Chang LC APOBEC3B: A potential factor suppressing growth of human hepatocellular carcinoma cells. Anticancer Res 35: 1521-1527, 2015.

28. Periyasamy M, Singh AK, Gemma C, Kranjec C, Farzan R, Leach DA, Navaratnam N, Pálinkás HL, Vértessy BG, Fenton TR, et al: p53 controls expression of the DNA deaminase APOBEC3B to limit its potential mutagenic activity in cancer cells. Nucleic Acids Res 45: 11056-11069, 2017.

29. Gomez-Manzano C, Fueyo J, Kyritsis AP, Steck PA, Roth JA, Mcdonnell TJ, Steck KD, Levin VA and Yung WK: Adenovirus-mediated transfer of the $p 53$ gene produces rapid and generalized death of human glioma cells via apoptosis. Cancer Res 56: 694-699, 1996.

30. Van Meir EG, Kikuchi T, Tada M, Li H, Diserens AC, Wojcik BE, Huang HJ, Friedmann T, de Tribolet $\mathrm{N}$ and Cavenee WK: Analysis of the $p 53$ gene and its expression in human glioblastoma cells. Cancer Res 54: 649-652, 1994.
31. Chou WC, Chen WT, Hsiung CN, Hu LY, Yu CJ, Hsu HM and Shen CJ: B-Myb induces APOBEC $3 B$ expression leading to somatic mutation in multiple cancers. Sci Rep 7: 44089, 2017.

32. Maruyama W, Shirakawa K, Matsui H, Matsumoto T, Yamazaki H, Sarca AD, Kazuma Y, Kobayashi M, Shindo K and Takaori-Kondo A: Classical NF- $\kappa \mathrm{B}$ pathway is responsible for APOBEC3B expression in cancer cells. Biochem Biophys Res Comm 478: 1466-1471, 2016.

33. Leonard B, McCann JL, Starrett GJ, Kosyakovsky L, Luengas EM, Molan AM, Burns MB, McDougle RM, Parker PJ, Brown WL, et al: The $\mathrm{PKC} / \mathrm{NF}-\mathrm{kB}$ signaling pathway induces APOBEC3B expression in multiple human cancers. Cancer Res 75: 4538-4547, 2015.

34. Nikkilä J, Kumar R, Campbell J, Brandsma I, Pemberton HN, Wallberg F, Nagy K, Scheer I, Vertessy BG, Serebrenik AA, et al: Elevated APOBEC3B expression drives a kataegic-like mutation signature and replication stress-related therapeutic vulnerabilities in p53-defective cells. Br J Cancer 117: 113-123, 2017. 